\title{
Robust static output-feedback controller design against sensor failure for vehicle dynamics
}

\author{
Sabrina Aouaouda ${ }^{1}$, Mohammed Chadli ${ }^{2}$, Hamid-Reza Karimi ${ }^{3}$ \\ ${ }^{1}$ LEER Laboratory, Faculty of Engineering and Science, University of Souk Ahras, BP 1553, 41000 Souk Ahras, Algeria \\ 2 University of Picardie Jules Verne, MIS (E.A.4290), 33, rue Saint-Leu, 80039 Amiens, France \\ ${ }^{3}$ Department of Engineering, Faculty of Engineering and Science, University of Agder, N-4879 Grimstad, Norway \\ E-mail: saouaouda@yahoo.fr
}

Received on 15 August 2013

Revised on 25 March 2014

Accepted on 21 April 2014

\section{Introduction}

In the last decades, enormous efforts have been devoted in developing intelligent systems for road vehicles. Thus, a trend was the application of active safety systems to improve vehicle handling characteristics like stability and comfort. Various works have been carried on collision warning, collision avoidance, adaptive cruise control and automated lane-keeping systems. Furthermore, majority of cars are nowadays equipped with traction control system, anti-lock braking system and many variants of electronic stability program [1]. However, the development of effective control systems in more challenging operating conditions and systems failure is still the objective of intense research from both academic and industrial perspectives. In terms of the translation vehicle motion, three types of control systems for vehicle dynamics can be distinguished: lateral, longitudinal and vertical control system. The presented work focuses on fault-tolerant control of vehicle lateral dynamics. Hence, an occurring fault must not only be detected and isolated but also accommodated by a so-called fault-tolerant control law, to preserve satisfactory system performances.

Over the past decades, fault diagnosis and fault-tolerant control strategies (FTC) have been proposed especially for sensor and/or actuator faults for vehicle lateral dynamics [2-4]. Two classes of the existing strategies have been distinguished. The first one is the so-called passive FTC or robust control where faults are treated as non-structural bounded uncertainties [5-7]. However, the issues of fault detection and estimation are not involved either. Contrarily to the passive FTC, active FTC requires the knowledge of the faults to reconfigure the controller law to maintain system stability, thus ensuring a smooth operation (see e.g.
[8-12] and references therein). The success of the previous methods mainly depends on the model complexity. Indeed, most studies have considered simple models and generally linear. The reality is far from these assumptions and systems are extremely non-linear [10]. Moreover, a large class of non-linear systems can be well approximated by TakagiSugeno (T-S) models $[10,11,13]$. This later is described by a set of linear time invariant (LTI) models and an interpolation mechanism between these models based on non-linear weighting functions. Indeed, the $\mathrm{T}-\mathrm{S}$ models can be cast into two main classes depending on whether the decision variables are measurable or not [14]. Furthermore, the T-S models with unmeasurable premise variables may be more interesting [14, 15]. Recently, it has been demonstrated that the multiple model approach is suitable for observer and/or controller design, because it allows avoiding the need of Lipschitz hypothesis like [16, 17] does.

Observers design for the non-linear $\mathrm{T}-\mathrm{S}$ systems has been studied using a quadratic Lyapunov functions (see e.g. $[18,19]$ and references therein). These approaches remain conservative since a common Lyapunov matrix must be found for a set of linear matrix inequalities (LMIs) [20]. To leave the quadratic framework, some works deal with polyquadratic and non-quadratic approaches [21-24] for control and observer or filter design [14, 21, 25-27]. In this context, relaxation schemes have been proposed for fault diagnosis and fault-tolerant control [6, 11, 15, 28-31], and even for Markovian jump systems with sensor saturation [32] and network sensors [33-35].

Among control theory, regarding to output stabilisation of $\mathrm{T}-\mathrm{S}$ models, several techniques have been proposed for dynamic output-feedback FTC design [2, 36, 37]. In these studies, $\mathrm{T}-\mathrm{S}$ systems with sensor failures are considered in 
which an augmented fault observer is designed to yield fault estimates and, based on the on-line fault estimates, observerbased static output-feedback fault-tolerant controllers are proposed. Following this way, one of our preliminary studies has dealt with the problem of robust sensor fault detection observer design for non-linear T-S systems with unmeasurable premise variables [38]. In the later, in aid of the descriptor approach, a mixed $H_{-} / H_{\infty}$ observer design for nominal $\mathrm{T}-\mathrm{S}$ systems has been proposed. However, this preliminary result discusses only sensor fault estimation or detection without considering the problem of fault-tolerant controller design. However, it is noted that complementary works have been recently done in the discrete-time framework, which is reputed more favourable than the continuous-time case for LMI purposes. Motivated by the issues mentioned above, this paper further investigates the issues of descriptor approach and multiple Lyapunov functions leading to strict LMIs based on robust fault estimation and fault-tolerant controllers design for the class of continuous-time disturbed $\mathrm{T}-\mathrm{S}$ systems. Taking advantages of the descriptor representation, it will be emphasised through a vehicle non-linear model how this modelling approach is relevant to deal with unmeasured premise variables for FTC design. Moreover, the non-quadratic framework will be considered leading to less conservative LMI conditions [39].

This paper is organised as follows. First, in Section 2, the studied vehicle lateral dynamics model and the problem statement are defined with highlights on the descriptor approach and unmeasurable premises. Then, Section 3 presents the robust observer-based FTC strategy: observer and controller gains are designed using relaxed nonquadratic LMI-based robust SOFC design conditions. Finally, vehicle lateral dynamics model is provided to show the effectiveness of the proposed approach.

Notation. Throughout this paper, when there is no ambiguity, the time $\mathrm{t}$ in a time varying variable will be omitted for space convenience. As usual, in a matrix, $(*)$ denotes a symmetrical transpose quantity. $\mathcal{H}(\mathfrak{H})$ denotes the Hermitian of the matrix $\mathfrak{H}$, that is, $\mathcal{H}(\mathfrak{H})=\mathfrak{H}+\mathfrak{H}^{\mathrm{T}}$. Moreover $\mathrm{I}_{n}$ is the identity matrix with appropriate dimensions.

\section{System modelling and problem formulation}

A schematic diagram of the considered vehicle model is shown in Fig. 1. The system under consideration describes vehicle lateral dynamics, which is obtained by considering the well-known single-track (bicycle) model with a roll degree of freedom, and mainly treated as a multi-model system in $[1,3,4]$. First the vehicle non-linear system with the multimodel representation used is detailed.

\subsection{Takagi-Sugeno vehicle model}

In this paper, a two-dimensional non-linear model of vehicle with non-linear tire characteristics can be described by the

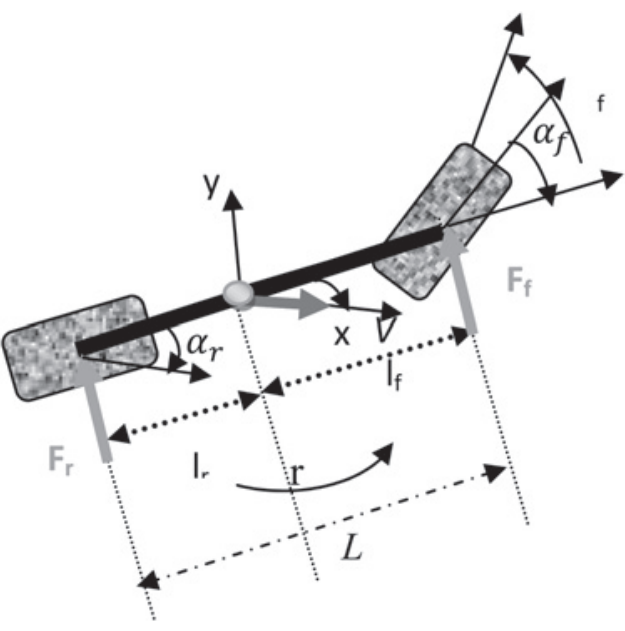

Fig. 1 Schematic diagram of the bicycle model

following differential equations [1, 3, 4]

$$
\left[\begin{array}{c}
\dot{\beta} \\
\dot{r}
\end{array}\right]=\left[\begin{array}{c}
\frac{F_{\mathrm{f}}+F_{\mathrm{r}}}{m U} \\
\frac{a_{\mathrm{f}} F_{\mathrm{f}}-a_{\mathrm{r}} F_{\mathrm{r}}}{J}+\frac{M}{J}
\end{array}\right]
$$

where, $F_{\mathrm{f}}$ is the cornering force of the two front tires, $F_{\mathrm{r}}$ is the cornering force of the two rear tires, $\beta$ denotes the sideslip angle and $r$ is the yaw velocity. $U$ is the vehicle velocity, $J$ is the yaw moment of inertia, $M$ is the yaw moment and $m$ is the vehicle mass.

Cornering forces $F_{\mathrm{f}}$ and $F_{\mathrm{r}}$, based on the magic formula [1], are given as functions of tire slip angles by the following expressions (see (2))

with

$$
\begin{aligned}
& \alpha_{\mathrm{f}}=\delta_{\mathrm{f}}-\beta-\tan ^{-1}\left(\frac{a_{\mathrm{f}}}{U} r \cos (\beta)\right), \\
& \alpha_{\mathrm{r}}=\tan ^{-1}\left(\frac{a_{\mathrm{r}}}{U} r \cos (\beta)\right)-\beta
\end{aligned}
$$

where $\delta_{\mathrm{f}}$ is the front steer angle, $a_{\mathrm{f}}$ is the slip angle of the front tires and $a_{\mathrm{r}}$ is the slip angle of the rear tires (Fig. 1). Coefficients $D_{i}, L_{i}, G_{i}$ and $V_{i}(i=\mathrm{f}, \mathrm{r})$ depend on the tire characteristics, road adhesion coefficient and the vehicle operational conditions. Generally, in control design, only the linear part of the forces is considered. However, this approximation is only valid for low slip angle. In this work, using $\mathrm{T}-\mathrm{S}$ fuzzy representation, we have approximated non-linear behaviour of forces (2) by two slip regions $M_{1}$ and $M_{2}$. We obtain two rules defined as follows

if $\left|\alpha_{\mathrm{f}}\right|$ is $M_{1}$ then

$$
\left\{\begin{array}{l}
F_{\mathrm{f}}=C_{\mathrm{f} 1}(\sigma) \alpha_{\mathrm{f}} \\
F_{\mathrm{r}}=C_{\mathrm{r} 1}(\sigma) \alpha_{\mathrm{r}}
\end{array}\right.
$$

if $\left|\alpha_{\mathrm{f}}\right|$ is $M_{2}$ then

$$
\left\{\begin{array}{l}
F_{\mathrm{f}}=C_{\mathrm{f} 2}(\sigma) \alpha_{\mathrm{f}} \\
F_{\mathrm{r}}=C_{\mathrm{r} 2}(\sigma) \alpha_{\mathrm{r}}
\end{array}\right.
$$

$$
\begin{aligned}
& F_{\mathrm{f}}=D_{\mathrm{f}}(\sigma) \sin \left[L_{\mathrm{f}}(\sigma) \tan ^{-1}\left\{G_{\mathrm{f}}(\sigma)\left(1-V_{\mathrm{f}}(\sigma)\right) \alpha_{\mathrm{f}}+V_{\mathrm{f}}(\sigma) \tan ^{-1}\left(G_{\mathrm{f}}(\sigma) \alpha_{\mathrm{f}}\right)\right\}\right] \\
& F_{\mathrm{r}}=D_{\mathrm{r}}(\sigma) \sin \left[L_{\mathrm{r}}(\sigma) \tan ^{-1}\left\{G_{\mathrm{r}}(\sigma)\left(1-V_{\mathrm{r}}(\sigma)\right) \alpha_{\mathrm{r}}+V_{\mathrm{r}}(\sigma) \tan ^{-1}\left(G_{\mathrm{r}}(\sigma) \alpha_{\mathrm{r}}\right)\right\}\right]
\end{aligned}
$$


where $C_{\mathrm{f} i}$, and $C_{\mathrm{r} i}$ represent front and rear tire stiffness which depend on the road adhesion and the mass of vehicle. The overall forces are then obtained by

$$
\left\{\begin{array}{l}
F_{\mathrm{f}}=\mu_{1}\left(\left|\alpha_{\mathrm{f}}\right|\right) C_{\mathrm{f} 1}(\sigma) \alpha_{\mathrm{f}}+\mu_{2}\left(\left|\alpha_{\mathrm{f}}\right|\right) C_{\mathrm{f} 2}(\sigma) \alpha_{\mathrm{f}} \\
F_{\mathrm{r}}=\mu_{1}\left(\left|\alpha_{\mathrm{f}}\right|\right) C_{\mathrm{r} 1}(\sigma) \alpha_{\mathrm{r}}+\mu_{2}\left(\left|\alpha_{\mathrm{f}}\right|\right) C_{\mathrm{r} 2}(\sigma) \alpha_{\mathrm{r}}
\end{array}\right.
$$

where $\mu_{i}, i=1,2$ is the $i$ th bell curve membership function of fuzzy set $M_{i}$ to be determined. It is important to note that the stiffness coefficients $C_{f i}$ and $C_{r i}$ are not constant and vary according to the road adhesion $\sigma$. To take into account these variations, we assume that these coefficients vary as follows

$$
\left\{\begin{array}{l}
C_{f i}=C_{f i 0}\left(1+d_{i} f_{i}\right) \\
C_{r i}=C_{r i 0}\left(1+d_{i} f_{i}\right)
\end{array} \quad\left|f_{i}\right| \leq 1\right.
$$

where $d_{i}$ indicates the deviation magnitude of the stiffness coefficient from its nominal value. The problem here consists in determining the parameters of the generalised Bell membership functions $\mu_{i}, i=1,2$ allowing to reproduce the non-linear behaviour of cornering forces. For this, we have developed an algorithm based on quadratic error minimisation which combines Levenberg-Marquadt algorithm and least squares method. We obtain

$$
\begin{gathered}
\mu_{i}\left(\left|\alpha_{\mathrm{f}}\right|\right)=\frac{\Delta_{i}\left(\left|\alpha_{\mathrm{f}}\right|\right)}{\sum_{i=1}^{2} \Delta_{i}\left(\left|\alpha_{\mathrm{f}}\right|\right)}, \Delta_{i}\left(\left|\alpha_{\mathrm{f}}\right|\right)=\frac{1}{\left[1+\left|\left(\left|\alpha_{\mathrm{f}}\right|-c_{i} / a_{i}\right)\right|\right]^{2 b_{i}}} \\
a_{1}=0.5077, \quad b_{1}=0.4748, \quad c_{1}=3.1893, \\
a_{2}=5.3907, \quad b_{2}=0.4356, \quad c_{2}=0.5633,
\end{gathered}
$$

Using the above approximation for non-linear lateral forces by $\mathrm{T}-\mathrm{S}$ fuzzy rules and by considering that

$$
\alpha_{\mathrm{f}} \cong \delta_{\mathrm{f}}-\beta-\frac{a_{\mathrm{f}} \dot{r}}{U}, \quad \alpha_{\mathrm{r}} \cong \frac{a_{\mathrm{r}} \dot{\psi}}{U}-\beta, \quad x=\left[\begin{array}{ll}
\beta & r
\end{array}\right]^{\mathrm{T}}
$$

non-linear model (1) with approximated forces (5) can be represented by the following T-S fuzzy model

$$
\left\{\begin{array}{l}
\dot{x}(t) \sum_{i=1}^{2} \mu_{i}\left(\left|\alpha_{\mathrm{f}}\right|\right)\left(A_{i}(\sigma) x(t)+B_{i}(\sigma) \delta_{\mathrm{f}}(t)+B M(t)\right) \\
y(t)=C x(t)
\end{array}\right.
$$

where (see equation at the bottom of the page)

The nominal values of the vehicle parameters are given in Table 1.

\subsection{Problem statement}

Generally, dynamic non-linear systems are modelled by the following ordinary differential equations

$$
\left\{\begin{array}{l}
\dot{x}(t)=f(x(t), u(t)) \\
y(t)=h(x(t), u(t))
\end{array}\right.
$$

Where $x(t) \in \mathbb{R}^{n}, \quad y(t) \in \mathbb{R}^{p}$, and $u(t) \in \mathbb{R}^{n_{u}}$ represent, respectively, the state vector, the measured system output
Table 1 Vehicle parameters

\begin{tabular}{lc}
\hline Parameters & Values \\
\hline$J$ & $3000 \mathrm{Kg} \mathrm{m}^{2}$ \\
$m$ & $1500 \mathrm{~m} \mathrm{Kg}$ \\
$a_{f}$ & $1.3 \mathrm{~m}$ \\
$a_{r}$ & $1.2 \mathrm{~m}$ \\
$U$ & $20 \mathrm{~m} / \mathrm{s}$ \\
$C_{f 10}$ & $60712 \mathrm{~N} / \mathrm{rad}$ \\
$C_{f 20}$ & $4812 \mathrm{~N} / \mathrm{rad}$ \\
$C_{r 10}$ & $60088 \mathrm{~N} / \mathrm{rad}$ \\
$C_{r 20}$ & $3455 \mathrm{~N} / \mathrm{rad}$ \\
\hline
\end{tabular}

and the bounded input vectors. The functions $f(x(t), u(t))$ and $h(x(t), u(t))$ are generally non-linear. As explained in the previous section, the $\mathrm{T}-\mathrm{S}$ formalism allows to represent any non-linear system in a compact set of the state-space with a convex combination of adequate $r$ linear submodels

$$
\left\{\begin{array}{l}
\dot{x}(t)=\sum_{i=1}^{r} \mu_{i}(\xi(t))\left(A_{i} x(t)+B_{i} u(t)\right) \\
y(t)=C x(t)
\end{array}\right.
$$

where $A_{i} \in \mathbb{R}^{n \times n}$ and $B_{i} \in \mathbb{R}^{n \times n_{u}}$. The activating functions $\mu_{i}(\xi(t))$ are non-linear that depend on the decision variable $\xi(t)$ which can be measurable (as the input or the output of the system) or non-measurable variables (as the state of the system) [15]. These functions verify the following so-called convex sum property

$$
\left\{\begin{array}{l}
0 \leq \mu_{i}(\xi(t)) \leq 1 \\
\sum_{i=1}^{r} \mu_{i}(\xi(t))=1 \forall i \in\{1,2, \ldots, r\}
\end{array}\right.
$$

The multiple model structure may be obtained either by identification, linearisation, or the so-called sector non-linearity transformation. In this work, the multiple model under study involves unmeasurable premise variables depending on the state variables. We consider the continuous-time $\mathrm{T}-\mathrm{S}$ model to be affected by sensor faults and unknown bounded disturbances. Then the T-S system (11) becomes

$$
\left\{\begin{array}{l}
\dot{x}(t)=\sum_{i=1}^{r} \mu_{i}(\xi(t))\left(A_{i} x(t)+B_{i} u(t)\right)+B_{d} d(t) \\
y(t)=C x(t)+D_{\mathrm{f}} f(t)
\end{array}\right.
$$

where $f(t) \in \mathbb{R}^{s}$ is the sensor fault vector and $d(t) \in \mathbb{R}^{n_{d}}$ is the unknown bounded disturbance vector. Matrices $B_{d}$ and $D_{\mathrm{f}}$ are of appropriate dimensions and $D_{\mathrm{f}}$ is assumed to be of

$$
A_{i}(\sigma)=\left[\begin{array}{cc}
-2 \frac{C_{\mathrm{f} i}(\sigma)+C_{\mathrm{r} i}(\sigma)}{m U} & -2 \frac{C_{\mathrm{f} i}(\sigma) a_{\mathrm{f}}-C_{\mathrm{r} i}(\sigma) a_{\mathrm{r}}}{m U^{2}}-1 \\
-2 \frac{C_{\mathrm{f} i}(\sigma) a_{\mathrm{f}}-C_{\mathrm{r} i}(\sigma) a_{\mathrm{r}}}{J} & -2 \frac{C_{\mathrm{f} i}(\sigma) a_{\mathrm{f}}^{2}+C_{\mathrm{r} i}(\sigma) a_{\mathrm{r}}^{2}}{J U}
\end{array}\right], \quad B_{i}(\sigma)=\left[\begin{array}{c}
\frac{2 C_{\mathrm{f} i}(\sigma)}{m u} \\
\frac{2 a_{\mathrm{f}} C_{\mathrm{f} i}(\sigma)}{J}
\end{array}\right], B_{i}=B=\left[\begin{array}{c}
0 \\
1 \\
\frac{1}{J}
\end{array}\right], C=[0
$$


full column rank. Construct the augmented descriptor system as follows

$$
\left\{\begin{array}{l}
\bar{E} \dot{\bar{x}}(t)=\sum_{i=1}^{r} \mu_{i}(\xi(t))\left(\bar{A}_{i} \bar{x}(t)+\bar{B}_{i} u(t)\right)+\bar{B}_{d} d(t)+\bar{D}_{h} h(t) \\
y(t)=\bar{C} \bar{x}(t)=C_{0} \bar{x}(t)+h(t)
\end{array}\right.
$$

where

$$
\begin{aligned}
\bar{x}(t) & =\left[\begin{array}{l}
x(t) \\
h(t)
\end{array}\right] \in \mathbb{R}^{n+p}, \quad \bar{E}=\left[\begin{array}{cc}
I_{n} & 0 \\
0 & 0
\end{array}\right], \bar{A}_{i}=\left[\begin{array}{cc}
A_{i} & 0 \\
0 & -I_{p}
\end{array}\right], \\
\bar{B}_{i} & =\left[\begin{array}{c}
B_{i} \\
0
\end{array}\right], \bar{B}_{d}=\left[\begin{array}{c}
B_{d} \\
0
\end{array}\right] \\
\bar{D}_{h} & =\left[\begin{array}{c}
0 \\
I_{p}
\end{array}\right], C_{0}=\left[\begin{array}{ll}
C & 0
\end{array}\right], \quad \bar{C}=\left[\begin{array}{ll}
C & I_{p}
\end{array}\right] \\
h(t) & =D_{f} f(t) \in \mathbb{R}^{p}
\end{aligned}
$$

To estimate both the system state and fault, the following singular observer structure is proposed

$$
\left\{\begin{array}{l}
E \dot{v}(t)=\sum_{i=1}^{r} \mu_{i}(\hat{\xi}(t))\left(S_{i} v(t)+\bar{B}_{i} u(t)\right) \\
\hat{\bar{x}}(t)=v(t)+L y(t) \\
\hat{y}(t)=C_{0} \hat{x}(t)=C \hat{x}(t)
\end{array}\right.
$$

where $v(t) \in \mathbb{R}^{n+p}$ is the auxiliary state vector of the observer and $\hat{\bar{x}}(t) \in \mathbb{R}^{n+p}$ is the estimate of $\bar{x}(t) . \hat{\xi}(t)$ is the unmeasured premise variable depending partially or completely on the estimated state $\hat{x}(t)$. The observer design is now reduced to finding the gains $S_{i}, E$ and $L$ such that the state and fault estimation error obey to a stable generating system.

\subsection{Descriptor observer design}

This section is devoted to the state and fault estimation of the continuous-time T-S model (13). In fact a more general situation will be analysed since sensor faults and unknown disturbances are envisaged. In this case, the observer design task consists in generating residual signals that are sensitive as possible to the faults, whereas insensitive to the disturbance so that the fault diagnosis is robust. The input signals $f(t)$ and $d(t)$ belong to $\mathcal{L}_{2}$ set. The $\mathcal{L}_{2}$-norm of a signal $H(t) \in \mathcal{L}_{2}$ is defined by

$$
\|H(t)\|_{2}=\left(\int_{0}^{+\infty} H^{\mathrm{T}}(t) H(t) \mathrm{d} t\right)^{1 / 2}
$$

In fact, the problem of residual generation can be viewed as $\mathcal{L}_{2}$-control (i.e. generalisation of $H_{\infty}$-control problem to the non-linear case), since the residual generator is designed by minimising the $\mathcal{L}_{2}$-gain from the disturbance signals to the residual signal $r_{d}(t)=\sum_{i=1}^{r} \mu_{i}(\xi(t)) V_{i} C_{0} e_{d}(t)$. For this purpose, we propose the following theorem.

Theorem 1: The state observer in the form of $(15 \mathrm{a}-\mathrm{c})$ can asymptotically estimate states and sensor faults if there exist some symmetric positive definite matrices $P^{11}, P^{12}, P_{i}^{2}$, matrices $N_{1}, N_{2}$, and positive scalar $\lambda$ that minimise the scalar $\gamma$ under the following LMI constraints (see (17)) with

$$
\begin{aligned}
& \left\{\begin{array}{l}
\Delta_{1 j}=\mathcal{H}\left(P^{11} A_{j}\right)+\mathcal{H}\left(N_{1} C\right)+I_{n} \\
\Delta_{2 i j}=N_{1}^{\mathrm{T}}-P_{i}^{12 \mathrm{~T}} C A_{j}-N_{2} C
\end{array}\right. \\
& \left\{\begin{array}{l}
\tilde{A}_{i j}^{\mathrm{T}}=A_{i}^{\mathrm{T}}-A_{j}^{\mathrm{T}}, \tilde{B}_{i j}^{\mathrm{T}}=B_{i}^{\mathrm{T}}-B_{j}^{\mathrm{T}} \\
\mathcal{K}_{i j}=\left(B_{i}^{\mathrm{T}}-A_{j}^{\mathrm{T}}\right) P^{11}
\end{array}\right. \\
& \left\{\begin{array}{l}
N_{1}=P^{11} \Omega \wp^{-1} \\
N_{2}=P^{12}\left(\wp^{-1}+C \Omega \wp^{-1}\right)
\end{array}\right.
\end{aligned}
$$

The observer gains are then obtained by

$$
S_{j}=\left[\begin{array}{cc}
A_{j} & 0 \\
-C & -I_{p}
\end{array}\right], \quad L=\left[\begin{array}{c}
0 \\
I_{p}
\end{array}\right], \quad E=\left[\begin{array}{cc}
I_{n}+\Omega C & \Omega \\
\wp C & \wp
\end{array}\right]
$$

where $\Omega \in \mathbb{R}^{n \cdot p}$ and $\wp \in \mathbb{R}^{p \cdot p}$ are two free matrices chosen to obtain non-singular matrix $E$

$$
\begin{gathered}
\wp=\left(P_{12}^{-1} N_{2}-C P_{11}^{-1} N_{1}\right)^{-1} \\
\Omega=P_{11}^{-1} N_{1} \wp
\end{gathered}
$$

The attenuation level of exogenous disturbance signal on residuals is given by. Since $D_{\mathrm{f}}$ is assumed to be of full column rank, then the sensor faults estimation can be obtained by

$$
\hat{f}(t)=\left(D_{\mathrm{f}}^{\mathrm{T}} D_{\mathrm{f}}\right)^{-1} D_{\mathrm{f}}^{\mathrm{T}} \hat{h}(t)
$$

Proof: The proof can be inspired directly from [38].

\section{Remark 1:}

- To enhance the residual generator robustness with regard to faults $f(t)$ residual generator is designed to minimise the sensitivity to the perturbation and to maximise the sensitivity to faults $[15,38]$. In the case of multiple fault diagnosis, the optimisation problem can be resolved by introducing a residual generator for each fault. More details can be found in [15].

- Since $D_{\mathrm{f}}$ is assumed to be of full column rank, then the sensor faults estimation can be obtained by (22). This

$$
\left[\begin{array}{cccccc}
\Delta_{1 j} & * & * & * & * & * \\
\Delta_{2 i j} & -\mathcal{H}\left(N_{2}\right) & * & * & * & * \\
\tilde{A}_{i j}^{\mathrm{T}} P^{11} & -\tilde{A}_{i j}^{\mathrm{T}} C^{\mathrm{T}} P^{12} & \mathcal{H}\left(P_{i}^{2} A_{i}\right) & * & * & * \\
\mathcal{K}_{i j} & -\tilde{B}_{i j}^{\mathrm{T}} C^{\mathrm{T}} P^{12} & B_{i}^{\mathrm{T}} P_{i}^{2} & -\lambda^{2} I_{n_{u}} & * & * \\
B_{d}^{\mathrm{T}} P^{11} & -B_{d}^{\mathrm{T}} C^{\mathrm{T}} P^{12} & B_{d}^{\mathrm{T}} P_{i}^{2} & 0 & -\gamma^{2} I_{n_{d}} & * \\
V_{i} C & 0 & 0 & 0 & 0 & -I_{n}
\end{array}\right] \leq 0 \quad \text { for, } j=1, \ldots, r
$$




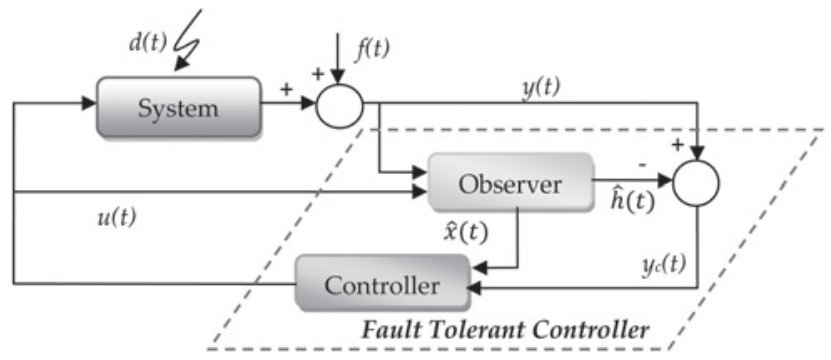

Fig. 2 Fault tolerant control scheme

assumption seems to be too strong in some cases, and the condition can be omitted using finite frequency domain method [40, 41].

\section{LMI-based conditions for FTC design}

In this section, to achieve the fault-tolerant control, a static output-feedback controller (SOFC) law is designed against sensor faults and external disturbances. As illustrated in Fig. 2, an observer provides faults and state estimations, required for the control law reconfiguration. Sufficient conditions to guarantee the stability of the resulting closed-loop system will be given in the form of LMIs.

Based on the parallel Disturbed Compensation (PDC) [42], a SOF control law is designed as follows

$$
u(t)=\sum_{i=1}^{r} \mu_{i}(\hat{\xi}(t)) K_{i} y_{\mathrm{c}}(t)
$$

where $K_{j}$ are the output-feedback gains to be determined and $y_{\mathrm{c}}(t)$ represents the compensated output defined by

$$
y_{\mathrm{c}}(t)=y(t)-\hat{h}(t)=y(t)-\ell \hat{\bar{x}}(t)
$$

with

$$
\ell=\left[\begin{array}{ll}
0 & I_{p}
\end{array}\right]
$$

Remark 2: Generally, the aim of SOFC is to control a dynamical system from only knowledge of measurable signals. Thus, the decision variables are required to depend only on the input $u(t)$, the output $y(t)$ and, eventually on measurable state variables. Whereas, it is still possible to design a robust static feedback controller in the case of unmeasurable premise variables. Indeed, in the sequel the structure of the FTC law is calculated, taking into account the states and sensor fault signals estimation derived from the descriptor observer $(15 \mathrm{a}-\mathrm{c})$.

Let us now analyse the stability of the closed-loop system. The state estimation error $e(t)=\bar{x}(t)-\hat{\bar{x}}(t)$ is generated using (20) by the following differential equation

$$
\begin{aligned}
E \dot{e}= & \sum_{i=1}^{r} \sum_{j=1}^{r} \mu_{i}(\xi) \mu_{j}(\hat{\xi})\left[\left(S_{j} L C_{0}+\bar{A}_{j}\right) e\right. \\
& \left.+\left(\bar{A}_{i}-\bar{A}_{j}\right) \bar{x}+\left(\bar{B}_{i}-\bar{B}_{j}\right) u+\bar{B}_{d} d\right]
\end{aligned}
$$

substituting (23a) into (24), we conclude that

$$
\begin{aligned}
E \dot{e}= & \sum_{i=1}^{r} \sum_{j=1}^{r} \mu_{i}(\xi) \mu_{j}(\hat{\xi})\left[S_{j} e+\left(\bar{A}_{i}-\bar{A}_{j}\right) \bar{x}\right. \\
& \left.+\left(\bar{B}_{i}-\bar{B}_{j}\right)\left(K_{j} \bar{c} \bar{x}-K_{j} \ell \overline{\hat{x}}\right)+\bar{B}_{d} d\right]
\end{aligned}
$$

Adding and subtracting $\left(\bar{B}_{i}-\bar{B}_{j}\right) K_{j} \ell \bar{x}(t),(25)$ can be rewritten as

$$
E \dot{e}=\sum_{i=1}^{r} \sum_{j=1}^{r} \mu_{i}(\xi) \mu_{j}(\hat{\xi})\left[\mathcal{F}_{i j} e+\mathcal{A}_{i j} \bar{x}+\bar{B}_{d} d\right]
$$

where

$$
\begin{gathered}
\mathcal{F}_{i j}=S_{j}+\left(\bar{B}_{i}-\bar{B}_{j}\right) K_{j} \ell \\
\mathcal{A}_{i j}=\left(\bar{A}_{i}-\bar{A}_{j}\right)+\left(\bar{B}_{i}-\bar{B}_{j}\right) K_{j}(\overline{\mathrm{C}}-\ell)
\end{gathered}
$$

Consequently (17) is equivalent to

$$
\dot{e}=\sum_{i=1}^{r} \sum_{j=1}^{r} \mu_{i}(\xi) \mu_{j}(\hat{\xi})\left[\overline{\mathcal{F}}_{i j} e+\overline{\mathcal{A}}_{i j}+G d\right]
$$

where the following nations are used (see (30) and (31))

To rewrite the whole model in a state-space representation, let us define the augmented state vector

$$
x_{a}^{\mathrm{T}}(t)=\left[e^{\mathrm{T}}(t) \quad x^{\mathrm{T}}(t)\right]
$$

The following closed-loop system is obtained

$$
\begin{aligned}
\dot{x}_{a}= & \sum_{i=1}^{r} \sum_{j=1}^{r} \mu_{i}(\xi) \mu_{j}(\hat{\xi}) \\
& \times\left\{\left[\begin{array}{cc}
\overline{\mathcal{F}}_{i j} & \overline{\mathcal{A}}_{i j} \\
0 & A_{i}+B_{i} K_{j} \mathrm{C}
\end{array}\right] x_{a}+\left[\begin{array}{c}
G \\
B_{d}
\end{array}\right] \dot{d}\right\}
\end{aligned}
$$

In the sequel, the following lemma is needed.

Lemma 1: Consider two real matrices $X, Y$ and $F(t)$ with appropriate dimensions, for any positive scalar $\delta$, the following inequality is verified

$$
X^{\mathrm{T}} F Y+Y^{\mathrm{T}} F^{\mathrm{T}} X \leq \delta X^{\mathrm{T}} X+\delta^{-1} Y^{\mathrm{T}} Y, \delta>0
$$

The objective is now to find out the observer and controller gains minimising the effect of the perturbation $d(t)$ on the

$$
\begin{gathered}
E^{-1}=\left[\begin{array}{cc}
I_{n} & -\Omega \wp^{-1} \\
-C & \wp^{-1}+C \Omega \wp^{-1}
\end{array}\right], \quad \overline{\mathcal{A}}_{i j}=\left[\begin{array}{c}
A_{i}-A_{j}+\left(B_{i}-B_{j}\right) K_{j} \mathrm{C} \\
-C\left(A_{i}-A_{j}\right)-C\left(B_{i}-B_{j}\right) K_{j} \mathrm{C}
\end{array}\right], \quad G=E^{-1} \bar{B}_{d}=\left[\begin{array}{c}
B_{d} \\
-C B_{d}
\end{array}\right] \\
\overline{\mathcal{F}}_{i j}=\left[\begin{array}{cc}
A_{j}+\Omega \wp^{-1} C & \Omega \wp^{-1}+\left(1+\Omega \wp^{-1} C\right)\left(B_{i}-B_{j}\right) K_{j} \\
-C A_{j}-\left(\wp^{-1}+C \Omega \wp^{-1}\right) C & -\left(\wp^{-1}+C \Omega \wp^{-1}\right)-\left(1+\wp^{-1}+C \Omega \wp^{-1}\right) C\left(B_{i}-B_{j}\right) K_{j}
\end{array}\right]
\end{gathered}
$$



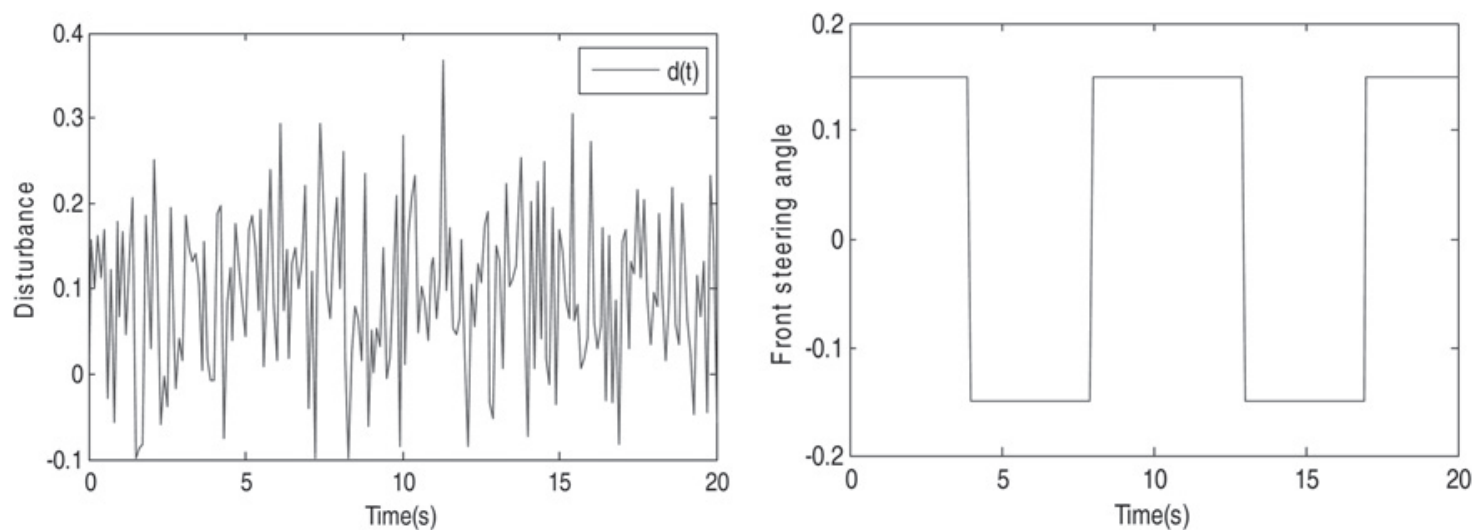

Fig. 3 Disturbance d(t) (left), front steering angle (right)

closed-loop system. That problem leads to solve a standard $\mathcal{L}_{2}$-control under the LMIs constraints provided by the following theorem.

Theorem 2: System (13) with observer-based controller $(15 \mathrm{a}-\mathrm{c})$ is asymptotically stable if there exist symmetric definite positive matrices $P_{11}, P_{12}, P_{2 i}$, matrices $Q_{1}, Q_{2}$ and positive scalars $\eta$, and $\delta_{i}, i=1, \ldots, 7$ satisfying the following conditions for, $i, j=1,2 \ldots, r$ and $i \neq j$

$$
\begin{gathered}
\min \eta \\
W_{i i}<0 \\
\frac{2}{r-1} W_{i i}+W_{i j}+W_{j i}<0
\end{gathered}
$$

where

$$
W_{i j}=\left[\begin{array}{cc}
\mathrm{Y}_{i j}^{(1,1)} & (*) \\
\mathrm{Y}_{i j}^{(2,1)} & \mathrm{Y}^{(2,2)}
\end{array}\right]
$$

with (see (38))

and

$$
\begin{aligned}
\mathrm{Y}^{(2,2)}= & -\operatorname{diag}\left(\delta_{1}+\delta_{5} \delta_{2}\left(\delta_{3}+\delta_{4}+\delta_{2}\right)^{-1}\right. \\
& \left.\times \delta_{3} \delta_{4} \delta_{1}^{-1} \delta_{6} \delta_{7}\left(\delta_{5}+\delta_{6}+\delta_{7}\right)^{-1}\right)
\end{aligned}
$$

$$
\left\{\begin{array}{l}
\hat{A}_{i j}^{\mathrm{T}}=A_{i}^{\mathrm{T}}-A_{j}^{\mathrm{T}} \\
\hat{B}_{i j}^{\mathrm{T}}=B_{i}^{\mathrm{T}}-B_{j}^{\mathrm{T}}
\end{array} ; \quad\left\{\begin{array}{l}
Q_{1}=P^{11} \Omega \wp^{-1} \\
Q_{2}=P^{12}\left(R^{-1}+C \Omega \wp^{-1}\right)
\end{array}\right.\right.
$$

The observer gains are then obtained by (20) $\Omega$ and $\wp$ are defined by

$$
\begin{gathered}
\wp=\left(P_{12}^{-1} Q_{2}-C P_{11}^{-1} Q_{1}\right)^{-1} \\
\Omega=P_{11}^{-1} Q_{1} \wp
\end{gathered}
$$

Proof: See Appendix 1

\section{Simulation results}

In this section, numerical simulations have been performed to demonstrate the effectiveness and the applicability of the proposed approach on the vehicle lateral dynamics model (1). The constructed T-S model, representing the vehicle model with premise variables depending on unmeasurable state variable, is used to build the observer. In the design, the vehicle parameters considered are given in Table 1.

Let us consider the following fault signal $f(t)=\left(f_{1}(t)\right.$, $\left.f_{2}(t)\right)^{T}$ affecting the system output behaviour and described as follows

$f_{1}(t)=0.3 \sin (t+2) \exp (t / 5)$ occurs at $3 \mathrm{~s} \leq t \leq 10 \mathrm{~s}$

$$
f_{2}(t)= \begin{cases}0.1(t-1) & 12 \mathrm{~s} \leq t<15 \mathrm{~s} \\ 0.01(t-1) & 15 \mathrm{~s} \leq t<18 \mathrm{~s} \\ 0 & \text { otherwise }\end{cases}
$$

Simulations were performed to assess the effects of external disturbance $d(t)$ and the efficiency of the $\mathcal{L}_{2}$-gain attenuation are illustrated in Fig. 3.

Recall that, only the yaw rate is supposed to be measurable with a gyroscope sensor. Moreover, the lateral velocity

$$
Y_{i j}^{(1,1)}=\left[\begin{array}{cccc}
\mathcal{H}\left(P_{11} A_{j}\right)+\mathcal{H}\left(Q_{1} C\right) & * & (*) & (*) \\
-P_{12} C A_{j}-Q_{2} C+Q_{1}^{\mathrm{T}} & -\mathcal{H}\left(Q_{2}\right) & * & * \\
\hat{A}_{i j}^{\mathrm{T}} P_{11} & -\hat{A}_{i j}^{\mathrm{T}} C^{\mathrm{T}} P_{21} & \mathcal{H}\left(P_{2 i}^{\mathrm{T}} A_{i}\right) & (*) \\
B_{d}^{\mathrm{T}} P_{11} & -B_{d}^{\mathrm{T}} C^{\mathrm{T}} P_{21} & B_{d}^{\mathrm{T}} P_{2 i} & -\eta^{2} I
\end{array}\right] ; \quad Y_{i j}^{(2,1)}=\left[\begin{array}{ccc}
0 & K_{j} \hat{B}_{i j} & 0 \\
0 & Q_{1} C & 0 \\
0 & Q_{2} & 0 \\
0 & K_{j} & 0 \\
0 & P_{21} C \hat{B}_{i j} & 0 \\
0 & 0 & P_{2 i} B_{i} \\
0 & 0 & K_{j} C
\end{array}\right]
$$



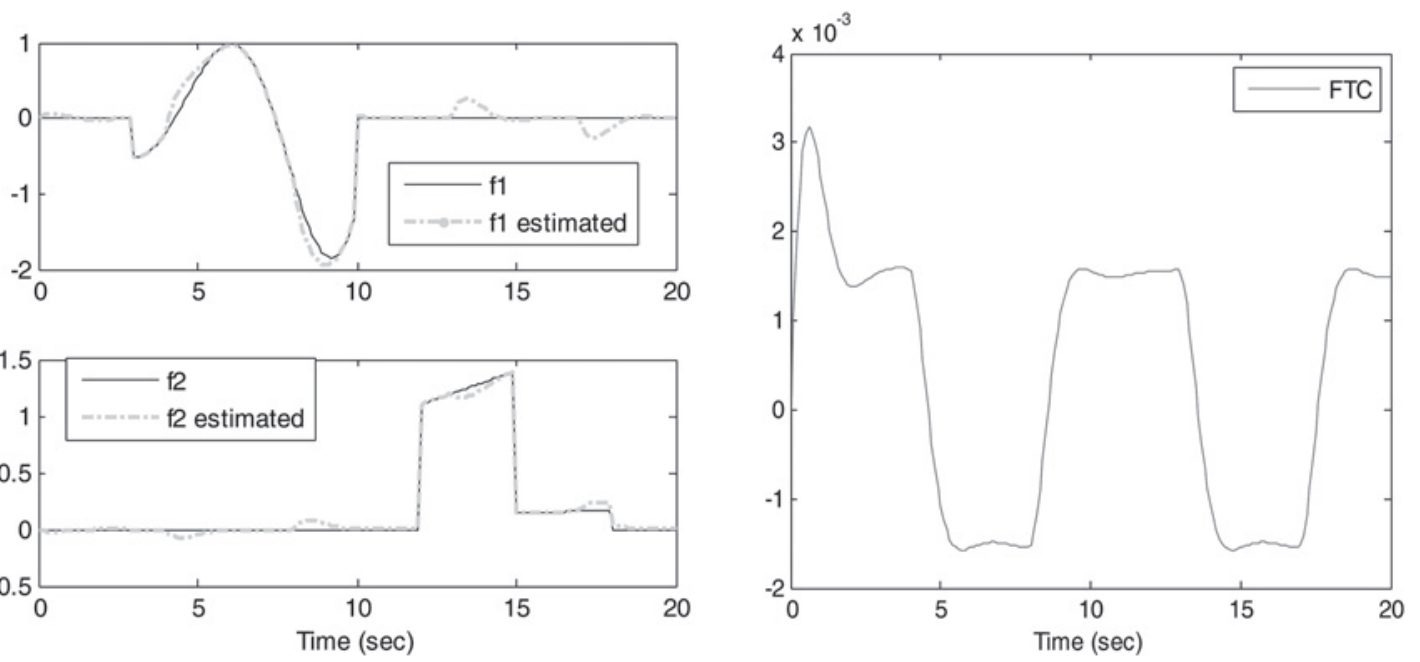

Fig. 4 Faults $\left(f_{1}, f_{2}\right)$ and their estimates (left), SOFC (right)
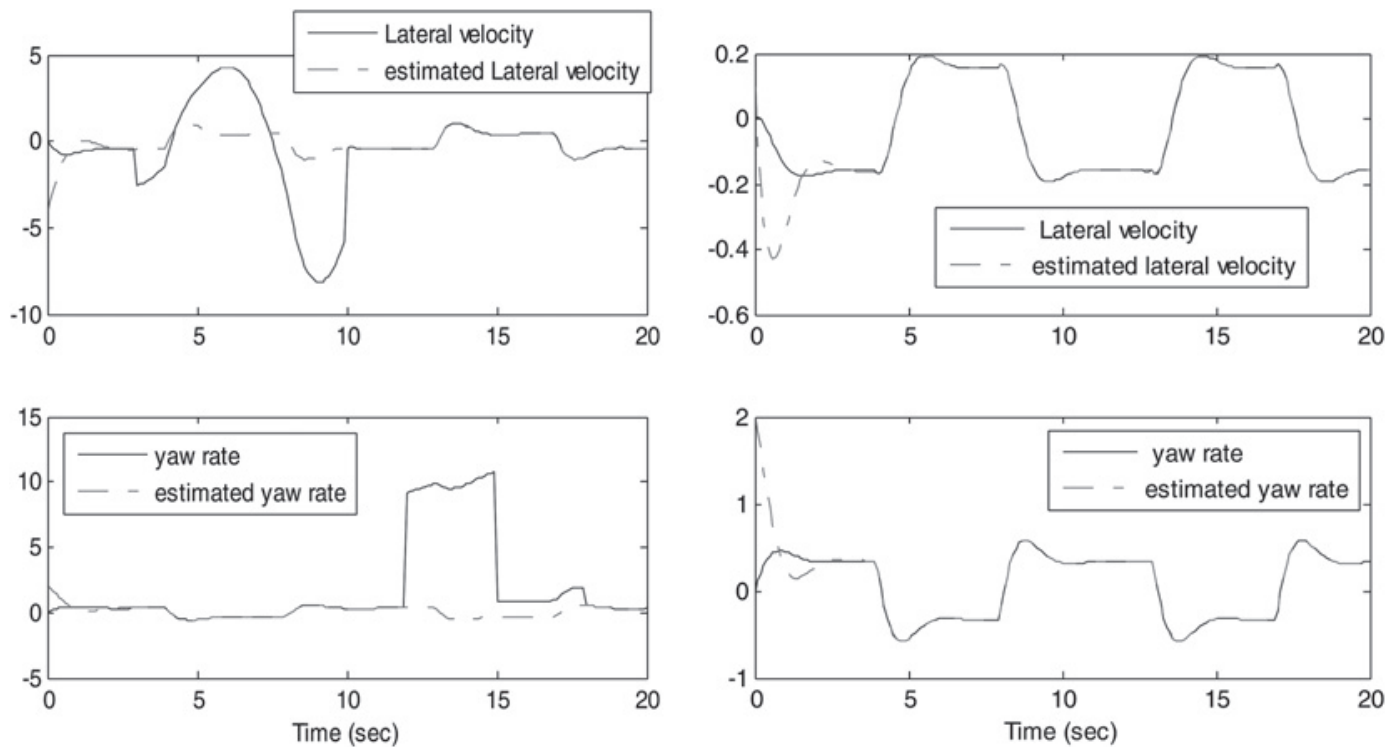

Fig. 5 Vehicle states without FTC strategy (left), with FTC strategy (right)
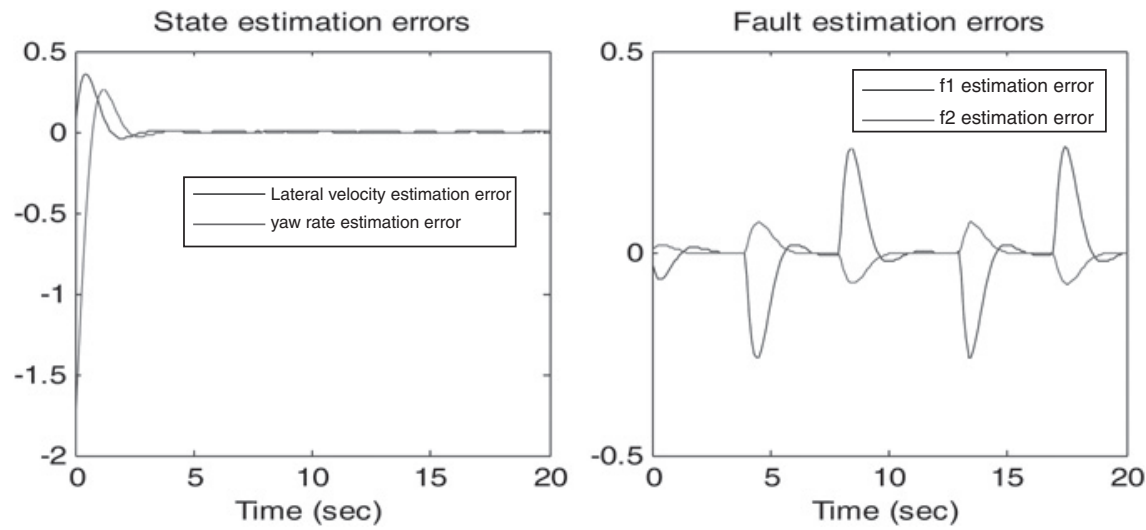

Fig. 6 State estimation errors (left), fault estimation errors (right) 
is estimated using the proposed observer. Solving the optimisation problem under LMI constraints in Theorem 2, results in the following observer and controller gain matrices for a nominal attenuation level $\eta=0.843$

$$
\begin{aligned}
& S_{1}=\left[\begin{array}{cccc}
-6.9425 & -0.8758 & 0 & 0 \\
26.8958 & -7.7952 & 0 & 0 \\
1.4062 & 1.9354 & -1 & 0 \\
0 & -1 & 0 & -1
\end{array}\right] \\
& S_{2}=\left[\begin{array}{cccc}
-0.4751 & -0.9968 & 0 & 0 \\
0.6997 & -0.4918 & 0 & 0 \\
1.4062 & 1.9354 & -1 & 0 \\
0 & -1 & 0 & -1
\end{array}\right] \text {, } \\
& L=\left[\begin{array}{llll}
0 & 0 & 1 & 0 \\
0 & 0 & 0 & 1
\end{array}\right]^{\mathrm{T}} \\
& K_{1}=\left[\begin{array}{ll}
-0.0019 & 0.0020
\end{array}\right], \quad K_{2}=\left[\begin{array}{ll}
-0.0020 & 0.0020
\end{array}\right] \\
& E=\left[\begin{array}{cccc}
0.8684 & 0.0066 & 0.0936 & 0.1878 \\
0.3121 & 0.9167 & -0.2219 & -0.5128 \\
-0.1232 & -0.0218 & 0.0876 & 0.1478 \\
-0.0420 & -0.0001 & 0.0299 & 0.0577
\end{array}\right]
\end{aligned}
$$

and

$$
\wp=\left[\begin{array}{ll}
0.0876 & 0.1478 \\
0.0299 & 0.0577
\end{array}\right], \quad \Omega=\left[\begin{array}{cc}
0.0936 & 0.1878 \\
-0.2219 & -0.5128
\end{array}\right]
$$

Fig. 4 shows the estimation of fault sensor and the obtained FTC control input. The state estimation errors together with the fault estimation errors are given in Fig. 6. The simulation results in Fig. 4 clearly demonstrate that the accurate estimates of the sensor fault signals are achieved via the proposed descriptor observer.

The simulation results are given in Fig. 5 with and without the FTC strategy. We can clearly see from the first case that the vehicle lost its performance just during the occurring of sensor faults. For the second case we can note that the vehicle remains stable despite the presence of faults and external disturbances, which shows the effectiveness of the proposed FTC strategy based on SOFC.

\section{Conclusion}

In this paper, the problem of robust fault-tolerant control method based on static output-feedback stabilisation for vehicle lateral dynamics model has been considered. First using $H_{\infty}$ formalism to derive conditions which ensure a minimal attenuation level of external disturbance, a descriptor state and fault estimation observer was designed to estimate state and fault signals. Then utilising the information of online fault estimates an observer-based static output-feedback fault-tolerant controller has been developed and its design has been involved through fuzzy Lyapunov approach. Sufficient conditions for the existence of both state observer and static output fault-tolerant controller were given in terms of LMIs. Finally, a non-linear model of the vehicle was presented to illustrate the effectiveness of the proposed approach when the sideslip angle is unavailable for measurement. It is noted that the extensions of the proposed model to take into account the parameter uncertainty or delay, which would help to calculate a more accurate results, deserve further investigation, see for instance [43-45]. Also, as a part of our future works, we will consider validation of the proposed theoretical results on vehicle system in a real traffic environment.

\section{Acknowledgments}

This work is partially supported in Norway by Section Innovation Norges Forskningsraad-Norwegian Research Council, and in France by the 'Ministère des Affaires étrangères' (MAE) and the 'ministère de l'Enseignement Supérieur et de la Recherche' (MESR) within the Aurora program. Authors to thank anonymous reviewers and the associate editor for their helpful comments and suggestions.

\section{References}

1 Lin, M., popov, M., Mcwilliam, S.: 'Stability and performance studies of driver-vehicle systems with electronic chassis control', Veh. Syst. Dyn., 2004, 41, (2), pp. 477-486

2 Gao, Z.F., Jiang, B., Qi, R.Y., Xu, Y.F., Cheng, Y.H.: 'Fuzzy observer design for near space vehicle with application to sensor fault estimation', ICIC Express Lett., 2010, 4, (1), pp. 177-182

3 Oudghiri, M., Chadli, M., El Hajjaji, A.: 'Robust observer-based fault tolerant control for vehicle lateral dynamics', Int. J. Veh. Des., 2008, 48, (3/4), pp. 173-189

4 Dahmani, H., Chadli, M., Rabhi, A., El Hajjaji, A.: 'Road curvature estimation for vehicle lane departure detection using a robust Takagi-Sugeno fuzzy observer'. Veh. Syst. Dyn., 2012 (doi:10.1080/00423114.2011.642806)

5 Zhang, K., Jiang, B., Staroswiecki, M.: 'Dynamic output feedbackfault tolerant controller design for Takagi-Sugeno fuzzy systems with actuator faults', IEEE Trans. Fuzzy. Syst., 2010, 18, (1), pp. 194-201

6 Blanke, M., Kinnaert, M., Lunze, J., Staroswiecki, M.: 'Diagnosis and Fault-Tolerant Control' (Springe-Verlag, Berlin, 2006)

7 Jiang, B., Staroswiecki, M., Cocquempot, V.: 'Fault accommodation for a class of nonlinear dynamic systems', IEEE Trans. Autom. Control, 2006, 51, pp. 1578-1583

8 Zhang, Y., Jiang, J.: 'Bibliographical review on reconfigurable faulttolerant control systems', Annu. Rev. Control, 2008, 32, pp. 229-252

9 Mufeed, M.M., Jiang, J., Zhang, Z.: 'Active fault tolerant control systems', Stochastic Analysis and Synthesis', (Springer, 2003)

10 Takagi, T., Sugeno, M.: 'Fuzzy identification of systems and its applications to modeling and control', IEEE Trans. Syst. Man Cybern., 1985, 15, pp. 116-132

11 Isermann, R.: 'Fault-Diagnosis Systems: An Introduction From Fault Detection to Fault Tolerance' (Springer, 2007)

12 Aouaouda, S., Chadli, M., Khadir, T., Bouarar, T.: 'Robust fault tolerant tracking controller design for unknown inputs $\mathrm{T}-\mathrm{S}$ models with unmeasurable premise variables', J. Process Control, 2012, 22, (5), pp. $861-872$

13 Marx, B., Koenig, D., Ragot, J.: 'Design of observers for TakagiSugeno descriptor systems with unknown inputs and application tofault diagnosis', IET Control Theory Appl., 2007, 1, (5), pp. 1487-1495

14 Yoneyama, J.: ' $\mathrm{H}_{\infty}$ filtering for fuzzy systems with immeasurable premise variables: an uncertain system approach', Fuzzy Sets Syst., 2009, 160, (12), pp. 1738-1748

15 Aouaouda, S., Chadli, M., Shi, P., Karimi, H.R.: 'Discrete-time $\mathrm{H}_{-} / \mathrm{H}_{\infty}$ sensor fault detection observer design for nonlinear systems with parameter uncertainty', Int. J. Robust Nonlinear Control, 2013, doi:10.1002/rnc.3089

16 Thau, F.E.: 'Observing the state of nonlinear systems', Int. J. Control, 1973, 17, pp. 471-479

17 Rajamani, R.: 'Observers for Lipschitz nonlinear systems', IEEE Trans. Autom. Control, 1998, 43, (3), pp. 397-401

18 Ding, B.C.: 'Quadratic boundedness via dynamic output feedback for constrained nonlinear systems in Takagi-Sugeno's form', Automatica, 2009, 45, (6), pp. 2093-2098

19 Sala, A., Ariño, C.: 'Asymptotically necessary and sufficient conditions for stability and performance in fuzzy control: applications of Pólya's theorem', Fuzzy Sets Syst., 2007, 158, (24), pp. 2671-2686

20 Boyd, S., El Ghaoui, L., Feron, E., Balakrishnan, V.: 'Linear matrix inequalities in system and control theory'. 15, SIAM Studies in Applied Mathematics, SIAM, Philadelphia, Pa, USA, 1994

21 Guerra, T.M., Kerkeni, H., Lauber, J., Vermeiren, l.: 'An efficient Lyapunov function for discrete TS models: observer design', IEEE Trans. Fuzzy Syst., 2012, 20, (1), pp. 187-192

22 Johansson, M., Rantzer, A., Arzén, K.E.: 'Piecewise quadratic stability of fuzzy systems', IEEE Trans. Fuzzy Syst., 1999, 7, (6), pp. 713-722

23 Tanaka, K., Hori, T., Wang, H.O.: 'A multiple Lyapunov function approach to stabilization of fuzzy control systems', IEEE Trans. Fuzzy Syst., 2003, 11, (4), pp. 582-589 
24 Chadli, M., Karimi, H.R.: 'Robust observer design for unknown inputs Takagi-Sugeno models', IEEE Trans. on Fuzzy Syst., 2013, 21, (1), pp. 158-164

25 Su, X., Shi, P., Wu, L., Song, Y.D.: 'A novel approach to filter design for T-S fuzzy discrete-time systems with timevarying delay', IEEE Trans. Fuzzy Syst., 2012, 20, (6), pp. 1114-1129

26 Gao, Z., Shi, X., Ding, S.X.: 'Fuzzy state-disturbance observer design for T-S fuzzy systems with application to sensor fault estimation', IEEE Trans. SMC B., 2008, 38, (3), pp. 875-880

27 Bouarar, T., Guelton, K., Manamanni, N.: 'Robust non-quadratic static output feedback controller design for Takagi-Sugeno systems using descriptor redundancy', Eng. Appl. Artif. Intell., 2013, 26, (2), pp. 739-756

28 Gertler, J.J.: 'Fault Detection and Diagnosis in Engineering Systems' (Marcel Dekker, 1998)

29 Ding, S.X.: 'Model-Based Fault Diagnosis Techniques Design Schemes, Algorithms and Tools' (Springer-Verlag, 2008)

30 Tanaka, K., Ohtake, H., Wang, H.O.: 'A descriptor system approach to fuzzy control system design via fuzzy Lyapunov functions', IEEE Trans. Fuzzy Syst., 2007, 15, (3), pp. 333-341

31 Karimi, H.R., Zapateiro, M., Luo, N.: 'A linear matrix inequality approach to robust fault detection filter design of linear systems with mixed time-varying delays and nonlinear perturbations', J. Franklin Inst., 2010, 347, pp. 957-973

32 Hongli, D., Ho, D.W.C., Gao, H.: 'Robust filtering for Markovian jump systems with randomly occurring nonlinearities and sensor saturation: the finite-horizon case', IEEE Trans. Signal Process., 2011, 59, (7), pp. 3048-3057

33 Hongli, D., Ho, D.W.C., Gao, H.: 'Distributed $\mathrm{H} \infty$ filtering for a class of Markovian jump nonlinear time-delay systems over lossy sensor networks', IEEE Trans. Ind. Electron., 2013, 60, (10), pp. $4665-4672$

34 Hongli, D., Ho, D.W.C., Gao, H.: 'Fault detection for Markovian jump systems with sensor saturations and randomly varying nonlinearities', IEEE Trans. Circuits Syst., I:Regul. Pap., 2012, 59, (10), pp. 2354-2362

35 Zhang, L., Boukas, E.-K., Baron, L., Karimi, H.R.: 'Fault detection for discrete-time markov jump linear systems with partially known transition probabilities', Int. J. Control, 2010, 83, (8), pp. $1564-1572$

36 Shi, S.H., Yuan, Z.H., Zhang, Q.L.: 'Fault-tolerant $\mathrm{H}_{\infty}$ filter design of a class of switched systems with sensor failures', Int. J. Innov. Comput. Inf. Control, 2009, 5, (11A), pp. 3827-3838

37 Lui, M., Shi, P.: 'Sensor fault estimation and tolerant control for Ito stochastic systems with simultaneous input and output disturbances', Automatica, 2013, 49, (5), pp. 1242-1250

38 Aouaouda, S., Chadli, M., Cocquempot, V., Khadir, M.T.: 'Multiobjective $\mathrm{H}_{-} / \mathrm{H}_{\infty}$ Fault detection observer design for TakagiSugeno fuzzy systems with unmeasurable premise variables: descriptor approach', Int. J. Adapt. Control Signal Process., 2013, 27, (12), pp. 1031-1047

39 Mozelli, L.A., Palhares, R.M. Avellar, G.S.C. 'A systematic approach to improve multiple Lyapunov function stability and stabilization conditions for fuzzy systems', Inf. Sci., 2009, 179, (8), pp. 1149-1162
40 Wang, H., Wang, J., Liu, J., Lam, J.: 'Iterative LMI approach for robust fault detection observer design'. Proc. of the 42nd IEEE Conf. on Decision And Control, Maui Hawaii, USA, December 2003

41 Gertler, J.J., Kunwer, M.M.: 'Optimal residual decoupling for robust fault diagnosis', Int. J. Control, 1995, 61, (2), pp. 395-421

42 Tanaka, K., Wang, H.O.: 'Fuzzy control systems design and analysis. a linear matrix inequality approach' (Wiley, New York, USA, 2001)

43 Li, H., Jing, X., Karimi, H.R.: 'Output-feedback based Hळcontrol for active suspension systems with control delay', IEEE Trans. Ind. Electron., 2014, 61, (1), pp. 436-446

44 Karimi, H.R.: 'Robust delay-dependent H $\infty$ control of uncertain Markovian jump systems with mixed neutral, discrete and distributed time-delays', IEEE Trans. Circuits Syst. I, 2011, 58, (8), pp. 1910 1923

45 Chadli, M., Guerra, T.M.: 'LMI solution for robust static output feedback control of Takagi-Sugeno fuzzy models', IEEE Trans. Fuzzy Syst., 2012, 20, (6), pp. 1160-1165

46 Mozelli, L.A., Palhares, R.M.: 'Less conservative $\mathrm{H} \infty$ fuzzy control for discrete-time Takagi-Sugeno Systems', Math. Prob. Eng., 2011, 2011, Article ID 361640

47 Zhou, K., Khargonekar, P.P.: 'Robust stabilization of linear systems with norm-bounded time-varying uncertainty', Syst. Control Lett., 1988, 10, pp. 17-20

\section{Appendix}

\subsection{Appendix 1: Proof of Theorem 2}

Proof: To obtain less conservative conditions, the following non-quadratic Lyapunov function [46] is adopted

$$
V(e(t), x(t))=\left[\begin{array}{l}
\dot{e}(t) \\
\dot{x}(t)
\end{array}\right]^{T}\left(\sum_{i=1}^{r} \mu_{i}(\xi(t)) \mathcal{P}_{i}\right)\left[\begin{array}{l}
e(t) \\
x(t)
\end{array}\right]
$$

where $\mathcal{P}_{i}=\operatorname{diag}\left[P_{1} \quad P_{2 i}\right]$ and $P_{1}, P_{2 i}$ are symmetric positive definite matrices. The closed-loop system with the FTC control is stable and the $\mathcal{L}_{2}$-gain from $d(t)$ to $e(t)$ is bounded by $\eta$ if $\dot{V}\left(x_{a}(t)\right)+e^{\mathrm{T}}(t) e(t)-\eta^{2} d^{\mathrm{T}}(t) d(t)<0$. The derivative of the Lyapunov function $V\left(x_{a}(t)\right)$ is expressed as (see (A.2))

This condition is negative definite if (see (A.3)) where (see (A.4))

Indeed, after some manipulation the inequality (A.3) becomes (see (A.5))

$$
\begin{aligned}
& \dot{V}\left(x_{a}\right)=\sum_{i=1}^{r} \sum_{j=1}^{r} \mu_{j}\left(\xi_{f}\right) \mu_{j}\left(\hat{\xi}_{f}\right) x_{a}^{\mathrm{T}} \mathcal{H}\left(\mathcal{P}_{i}^{\mathrm{T}}\left[\begin{array}{cc}
\overline{\mathcal{F}}_{i j} & \overline{\mathcal{A}}_{i j} \\
0 & A_{i}+B_{i} K_{j} C
\end{array}\right]\right) x_{a}+\mathcal{H}\left(x_{a}^{\mathrm{T}} \mathcal{P}_{i}\left[\begin{array}{c}
G \\
B_{d}
\end{array}\right] d\right) \\
& \sum_{i=1}^{r} \sum_{j=1}^{r} \mu_{i}\left(\xi_{f}\right) \mu_{j}\left(\hat{\xi}_{f}\right)\left[\begin{array}{ccc}
\Xi_{i j} & (*) & (*) \\
\overline{\mathcal{A}}_{i j}^{\mathrm{T}} P_{1} & \mathcal{H}\left(P_{2 i}^{\mathrm{T}} A_{i}\right)+\mathcal{H}\left(P_{2 i}^{\mathrm{T}} B_{i} K_{j} C\right) & (*) \\
G^{\mathrm{T}} P_{1} & B_{d}^{\mathrm{T}} P_{2 i} & -\eta^{2} I
\end{array}\right]<0 \\
& \Xi_{i j}=\left[\begin{array}{cc}
\mathcal{H}\left(P_{11} A_{j}\right)+\mathcal{H}\left(Q_{1} C\right) & (*) \\
-P_{12} C A_{j}-Q_{2} C+Q_{1}^{\mathrm{T}}+K_{j}^{\mathrm{T}} \hat{B}_{i j}^{\mathrm{T}} P_{11}+K_{j}^{\mathrm{T}} \hat{B}_{i j}^{\mathrm{T}} C^{\mathrm{T}} Q_{1}^{\mathrm{T}} & -\mathcal{H}\left(Q_{2}\right)-\mathcal{H}\left(P_{12} C \hat{B}_{i j} K_{j}\right)-\mathcal{H}\left(Q_{2} \hat{B}_{i j} K_{j}\right)
\end{array}\right] \\
& \sum_{i=1}^{r} \sum_{j=1}^{r} \mu_{i}\left(\xi_{f}\right) \mu_{j}\left(\hat{\xi}_{f}\right)\left[\begin{array}{cccc}
\mathcal{H}\left(P_{11} A_{j}\right)+\mathcal{H}\left(Q_{1} C\right) & * & (*) & (*) \\
\Psi_{i j}^{12} & \Psi_{i j}^{22} & * & * \\
\Psi_{i j}^{13} & \Psi_{i j}^{23} & \Psi_{i j}^{33} & (*) \\
B_{d}^{T} P_{11} & -B_{d}^{T} C^{T} P_{21} & B_{d}^{T} P_{2 i} & -\eta^{2} I
\end{array}\right]<0
\end{aligned}
$$


where

$$
\begin{gathered}
\Psi_{i j}^{12}=P_{12} C A_{j}-Q_{2} C+Q_{1}^{\mathrm{T}}+K_{j}^{\mathrm{T}} \hat{B}_{i j}^{\mathrm{T}} P_{11}+K_{j}^{\mathrm{T}} \hat{B}_{i j}^{\mathrm{T}} C^{\mathrm{T}} Q_{1}^{\mathrm{T}} \\
\Psi_{i j}^{13}=\hat{A}_{i j}^{\mathrm{T}} P_{11}+C^{\mathrm{T}} K_{j}^{\mathrm{T}} \hat{B}_{i j}^{\mathrm{T}} P_{11} \\
\Psi_{i j}^{23}=-\hat{A}_{i j}^{\mathrm{T}} C^{\mathrm{T}} P_{21}-C^{\mathrm{T}} K_{j}^{\mathrm{T}} \hat{B}_{i j}^{\mathrm{T}} C^{\mathrm{T}} P_{21} \\
\Psi_{i j}^{22}=-\mathcal{H}\left(Q_{2}\right)-\mathcal{H}\left(P_{12} C \hat{B}_{i j} K_{j}\right)-\mathcal{H}\left(Q_{2} \hat{B}_{i j} K_{j}\right) \\
\Psi_{i j}^{33}=\mathcal{H}\left(P_{2 i}^{\mathrm{T}} A_{i}\right)+\mathcal{H}\left(P_{2 i}^{\mathrm{T}} B_{i} K_{j} \mathrm{C}\right)
\end{gathered}
$$

Using the well-known Lemma 1, there exist positive scalars $\delta_{i}, i=1, \ldots, 7$ such that (see (A.6)) where

$$
\begin{aligned}
\Xi_{i j}^{11}= & \mathcal{H}\left(P_{11} A_{j}\right)+\mathcal{H}\left(Q_{1} C\right)+\left(\delta_{1}^{-1}+\delta_{5}^{-1}\right) P_{11} \hat{B}_{i j} \hat{B}_{i j}^{\mathrm{T}} P_{11} \\
& +\delta_{2}^{-1} Q_{1} C C^{\mathrm{T}} Q_{1}^{\mathrm{T}} \\
\Xi_{i j}^{22}= & -\mathcal{H}\left(Q_{2}\right)+\left(\delta_{3}+\delta_{2}+\delta_{4}\right) K_{j}^{\mathrm{T}} \hat{B}_{i j}^{\mathrm{T}} K_{j} \hat{B}_{i j}+\delta_{3}^{-1} Q_{1} C C^{\mathrm{T}} Q_{1}^{\mathrm{T}} \\
& +\delta_{4}^{-1} Q_{2}^{\mathrm{T}} Q_{2}+\delta_{1} K_{j}^{\mathrm{T}} K_{j}+\delta_{6}^{-1} P_{21} C \hat{B}_{i j} \hat{B}_{i j}^{\mathrm{T}} C^{\mathrm{T}} P_{21} \\
\Xi_{i j}^{33}= & \mathcal{H}\left(P_{2 i}^{\mathrm{T}} A_{i}\right)+\delta_{7}^{-1} P_{2 i} B_{i} B_{i}^{\mathrm{T}} P_{2 i}+\left(\delta_{5}+\delta_{6}+\delta_{7}\right) C^{\mathrm{T}} K_{j}^{\mathrm{T}} K_{j} C
\end{aligned}
$$

Applying Schur complement [47] on the BMI terms $\Xi_{i j}^{11}$, $\Xi_{i j}^{22}$ and $\Xi_{i j}^{33}$ the sufficient LMI conditions proposed in Theorem 2 hold.

$$
\sum_{i=1}^{r} \sum_{j=1}^{r} \mu_{i}\left(\xi_{f}\right) \mu_{j}\left(\hat{\xi}_{f}\right)\left[\begin{array}{cccc}
\Xi_{i j}^{11} & * & (*) & (*) \\
-P_{12} C A_{j}-Q_{2} C+Q_{1}^{\mathrm{T}} & \Xi_{i j}^{22} & * & * \\
\hat{A}_{i j}^{\mathrm{T}} P_{11} & -\hat{A}_{i j}^{\mathrm{T}} C^{\mathrm{T}} P_{21} & \Xi_{i j}^{33} & (*) \\
B_{d}^{\mathrm{T}} P_{11} & -B_{d}^{\mathrm{T}} C^{\mathrm{T}} P_{21} & B_{d}^{\mathrm{T}} P_{2 i} & -\eta^{2} I
\end{array}\right]<0
$$

